\title{
Reflexões sobre a comunicação e a aprendizagem como constituintes da inovação nas organizações
}

Reflections on communication and learning as parts of innovation in organizations

Reflexiones sobre la comunicación y el aprendizaje como componentes de la innovación en las organizaciones

Tainah Veras

- Doutoranda e Mestre em Comunicação na Faculdade de Arquitetura, Artes e Comunicação (Faac) da Unesp

- Membro do grupo de pesquisa CIG (Comunicação Organizacional; Inovação e Gestão), também da Faac/Unesp

- $\quad$ Especialista em Marketing e Comunicação pelo Instituto Nacional de Pós-Graduação (INPG)

- Bolsista da Agência Unesp de Inovação através do convênio com o Santander Universidades

- Professora das Faculdades Integradas de Bauru (FIB) no curso de Publicidade e Propaganda

- E-mail: tainah.veras@unesp.br

9.) Maria Eugênia Porém

- Pós-doutora em Comunicação pela ECA/USP

- Doutora em Educação e Mestre em Comunicação pela Unesp

- Especialista em Comunicação, Marketing e Negócios pela Universidade de Marília

- Professora assistente doutora da Universidade Estadual Paulista (Unesp), na Faculdade de Arquitetura, Artes e Comunicação (Faac)

- Líder do Grupo de Pesquisa Comunicação Organizacional, Inovação e Gestão (CIG).Bolsista CNPq, Modalidade/Categoria: EXP-SA-AS, no Programa Agentes Locais de Inovação (ALI) do Sebrae (2016-2017) e (2019/2020)

- E-mail: maria.porem@unesp.br 


\section{Resumo}

O ensaio visa refletir sobre a comunicação e a aprendizagem como constituintes da inovação nas organizações, enfocando maneiras de compreender o conceito para além de uma perspectiva meramente econômica e utilitarista. Entre os resultados encontrados, destaca-se a necessidade de estimular, coletivamente e por meio da comunicação, leituras de mundo mais conscientes e humanas sobre a inovação, que prezem pela capacidade das pessoas de transformarem a realidade na qual estão inseridas.

\section{PALAVRAS-CHAVES: INOVAÇÃO • COMUNICAÇÃO • APRENDIZAGEM・ORGANIZAÇÕES.}

\section{Abstract}

This article reflects on communication and learning as parts of innovation in organizations, focusing on ways to understand the concept beyond purely economic and utilitarian perspectives. Among the results found, we stress the need to collectively perform through communication more conscious and humane world understandings about innovation, valuing people's ability to transform the reality in which they live.

KEYWORDS: INNOVATION • COMMUNICATION • LEARNING • ORGANIZATIONS.

\section{Resumen}

El artículo tiene como objetivo reflexionar sobre la comunicación y el aprendizaje como componentes de la innovación en las organizaciones, centrándose en formas de comprender el concepto más allá de una perspectiva puramente económica y utilitaria. Entre los resultados encontrados, existe la necesidad de estimular colectivamente a través de la comunicación lecturas del mundo mas conscientes y humanas sobre la innovación, que valoran la capacidad de las personas para transformar la realidad en la que se insertan. 


\section{INTRODUÇÃO}

A

valorização exacerbada da inovação tornou-se recorrente na contemporaneidade, ao ponto de Sievers (2007) afirmar que as organizações ocidentais estão vivendo o fenômeno de neofilia, ou seja, do culto à novidade, que é contraposta ao passado, visto como antiquado.

Nessa mesma ordem, não é raro identificar ideias incorporadas como "verdades absolutas", nas quais a inovação é entendida como um conjunto de tecnologias viabilizadas pelos empreendedores e como fórmula para ampliar o desenvolvimento econômico, a competitividade e o lucro.

Estabelece-se assim um "regime de verdade no qual o valor do novo se associa menos ao progresso como emancipação social do que à utilidade e à venda para o mercado" (Diaz-Isenrath, 2008, p. 84).

Isso faz com que o conceito de inovação seja visto de forma limitada e instrumental, sem a criticidade reflexiva necessária. Como Fontenelle (2012, p.107), entendemos que lançar luz e problematizar essa realidade não significa "ser contra a inovação per se", mas sim expor "certo mal-estar diante de um discurso que parece tirar-nos a possibilidade de qualquer tipo de questionamento, tamanha a sua positividade".

Nesse sentido, destacamos que existem outras formas de compreender a inovação no contexto das organizações que extrapolam uma lógica positivista e simplificadora, e permitem vislumbrar o conceito como algo ligado à essência humana de pensar e viabilizar formas novas e melhores de agir (Fagerberg, 2003), construindo, significando e ressignificando experiências, relações, necessidades e mudanças do dia a dia. A nosso ver, é possível abordar essa perspectiva humana e social da inovação estabelecendo o nexo do conceito com a comunicação e a aprendizagem.

Com relação à comunicação, entendemos que ela está longe de ser um fenômeno secundário. Afinal, ela é condição essencial para a vida, e assim Serres (2003) menciona que poderíamos substituir a expressão "penso, logo existo" por "me religo, logo sou", reconhecendo o protagonismo inextrincável das relações na constituição de todos os processos e criações humanas, incluindo as organizações, que só existem "comunicacionalmente" (Marchiori, 2013).

Quanto à aprendizagem, buscando respaldo no contexto da educação a partir de Freire (1967), pontuamos que aprender demanda uma consciência crítica e questionadora para ir além das situações às quais se está condicionado, buscando integrar-se ao mundo, transformar a realidade, e não apenas acomodar-se às prescrições alheias.

Diante disso, esse artigo tem o objetivo de refletir sobre a comunicação e a aprendizagem como constituintes da inovação nas organizações, enfocando maneiras de compreender a referida inovação mais à frente de uma perspectiva meramente econômica e utilitarista, de forma que ela seja vista como meio para promover transformações, e não como fim em si.

Para tanto, estruturamos um ensaio no qual trazemos inicialmente contribuições de alguns autores sobre inovação em um prisma mais crítico; na sequência, apresentamos olhares sobre a comunicação e a aprendizagem, e, por fim, correlacionamos os enunciados dos autores em uma análise indutiva e interpretativa a fim de tecer reflexões sobre a temática. 


\section{TENSIONANDO O CONCEITO DE INOVAÇÃO}

A inovação costuma ser encarada como solução tecnológica inquestionável para propor novidades e alavancar a competitividade em diferentes contextos; porém, dificilmente há a preocupação em refletir porque o conceito possui esse status ufanista e se, historicamente, ele sempre foi valorizado dessa maneira.

Godin (2014) afirma que, apesar da exaltação da inovação na contemporaneidade, nos séculos XVII e XVIII, por exemplo, aqueles que buscavam inovar eram vistos como hereges, subversivos e inoportunos, ou seja, interessados em destruir a ordem estabelecida; somente a partir do século XIX a inovação passou a ser encarada de maneira positiva, sendo exaltada por conta de sua contribuição para o desenvolvimento econômico.

Dois fatores, especialmente, fizeram com que se consolidasse a visão progressista de inovação que prevalece até os dias atuais: a estruturação das indústrias, que acabou fomentando uma cultura de ampla valorização de produtos e novidades a partir da ótica capitalista; e o discurso de estudiosos que passaram a exaltar a importância da tecnologia e do protagonismo do empreendedor para criar soluções capazes de contribuir com o crescimento e a diferenciação das empresas (Godin, 2008).

Um desses estudiosos foi Schumpeter, considerado por muitos o "pai" da inovação. Segundo ele, o empreendedor deveria ter força de vontade para "arrancar, dentre o trabalho e a lida com as ocupações diárias, oportunidade e tempo para conceber e elaborar a combinação nova e resolver olhá-la como uma possibilidade real e não meramente como um sonho", o que "pressupõe um grande excedente de força sobre a demanda cotidiana e é algo peculiar e raro por natureza" (1997, p.93); ele atribui, assim, um caráter quase heroico a esse ator, distanciando-o consideravelmente dos demais indivíduos de uma organização.

Ainda de acordo com o pensamento desse autor, para empreender seria preciso assumir uma liderança econômica que privilegiasse a inovação e a diferenciasse da mera produção de invenções que não fossem levadas à prática. Adicionalmente, para Schumpeter (1997), o empreendedor deveria ser um líder empresarial perspicaz e enérgico focado em cumprir a tarefa específica de inovar, conduzindo os meios de produção para a real comercialização de novidades e impressionando as pessoas responsáveis por financiar economicamente as organizações.

Essa lógica de considerar quem está à frente do negócio de forma atomizada e calculista, e de vislumbrar a inovação como sinônimo de soluções comercializáveis, possui notoriedade hoje na maioria dos discursos dos governos, das organizações e das instituições midiáticas, e acaba por ignorar o contexto social em que cada empreendedor está inserido, fazendo com que aspectos humanos, culturais, relacionais e históricos sejam minimizados e silenciados.

Apesar dessa tendência que muitos atores têm em vislumbrar o conceito dessa forma utilitária, econômica e até mesmo egoísta da inovação, também existem estudiosos que possuem outro olhar para o fenômeno.

Badillo (2013), por exemplo, defende que as interações entre as pessoas, que são responsáveis por possibilitar combinações de ideias e conhecimentos distintos, são mais importantes do que o capital econômico mobilizado para inovar.

Por sua vez, Carneiro (2013) pontua: "Inovação é um meio, não um fim. É um COMO, não um O QUE". A perspectiva de Messina (2001) corrobora com essa ideia de inovação que vai além da busca por objetivos isolados, pois o autor afirma que o fenômeno é aberto, multidimensional, adota diferentes formas e significados de acordo com o contexto, e permite transformar o espaço em que ele ocorre. 
Entre as outras vertentes que encaram a inovação de maneira mais complexa, encontram-se ainda abordagens sobre a cultura de inovação, sendo uma delas preconizada por Jucevičius (2007; 2010).

Jucevičius (2007) destaca que, sabendo que a cultura costuma ser definida popularmente como "o jeito com que as coisas costumam ser feitas na empresa há algum tempo", a inovação envolve questionar as formas pré-estabelecidas de agir no dia a dia, abrindo espaço para ideias que desenvolvam novos valores, atitudes, rotinas e práticas na organização.

Para fazer isso, é preciso manter-se em estado constante de reflexão, curiosidade e abertura. Não à toa, Jucevičius (2010) afirma que toda cultura de inovação consiste em uma cultura de aprendizagem, e destaca que a cultura de inovação envolve um "questionamento permanente de seus próprios valores, crenças fundamentais e padrões de comportamento, ao mesmo tempo em que se mantém a capacidade de funcionar e produzir resultados inovadores" (Jucevičius, 2007, p.10, tradução nossa).

Entendemos que essa definição de cultura de inovação ligada ao questionamento se conecta de certa forma com a visão de Freire (1996) sobre a importância de realizar reflexões críticas no dia a dia, buscando, de forma contínua, conhecimentos que permitam a realização dessas reflexões; para o autor, o ser humano deve tomar consciência de que é um ser inacabado, que precisa se inserir em um movimento permanente de busca para aprender, ensinar, problematizar, dialogar e transformar a realidade de modo a fazer história, a produzir cultura e a estar verdadeiramente integrado ao ambiente com os demais.

Adotar uma postura questionadora e consciente como forma de buscar mudanças não significa abrir mão de padrões e rotinas comuns que alicerçam o cotidiano das organizações; o desafio é manter certas estruturas e, concomitantemente, tensionar as práticas que precisam ser modificadas para que ocorram processos de emancipação e integração dos seres humanos.

O delineamento de rotinas de reflexão sobre práticas culturais e a capacidade de compreender a inovação para além de um viés instrumental possuem relação direta, a nosso ver, com o entendimento do conceito como um fenômeno constituído pela comunicação e pela aprendizagem.

\section{OLHARES PARA A COMUNICAÇÃO E A APRENDIZAGEM}

D'Almeida e Andonova (2008, p.32) ressaltam que as primeiras abordagens sobre comunicação nas organizações privilegiaram atuações pontuais, unidimensionais e restritas, sem compreender o fenômeno comunicacional como protagonista desse universo e das práticas que o constituem.

Segundo as autoras, a comunicação se consolidou no âmbito profissional "a fim de atender às necessidades quase exclusivamente operacionais do nível micro-organizacional", como a resolução de problemas na execução de determinadas tarefas, a transmissão de informações e a harmonização de posicionamentos, com foco no aumento da eficiência e da produtividade.

Portanto, não houve a preocupação e a consciência iniciais de encarar os processos comunicacionais nas empresas de forma relacional, humana e socializadora, tampouco de estabelecer os imbricamentos necessários e inevitáveis da comunicação com outros processos, como a aprendizagem e a inovação.

Compreendemos que essa perspectiva instrumental, acessória e restritiva originalmente ligada à comunicação nas empresas deriva em grande parte da maneira simplista, totalizante, homogênea e hegemônica com que as próprias organizações são muitas vezes entendidas, ignorando o fato de que elas existem como um fenômeno vivo, heterogêneo, processual, relacional e múltiplo, que se estrutura em uma "ordem negociada entre cotidianos distintos" (Spink, 1996, p.9) viabilizados nas interações entre as pessoas. 
Segundo Stoffregen (2014), a valorização excessiva de procedimentos, artefatos e padrões para alcançar resultados faz com que sejam construídos discursos que tentam dar a impressão de que a empresa é uma entidade que existe por si só e é distinta do conjunto de seres humanos que a formam, ao invés de considerar "a dinâmica natural que leva as pessoas a priorizarem o contato humano em detrimento de qualquer normatividade ou padrão de eficiência" (Stoffregen, 2014, p.20, tradução nossa).

Quanto a isso, inclusive, Spink (1991) destaca que estudos feitos sobre a chegada de novas pessoas em uma empresa mostram que a maioria delas não se atenta aos conteúdos explicativos gerais compartilhados de forma prescritiva, e acaba preferindo conversar com alguém para perguntar como são as rotinas, quais são as normas e como é o dia a dia. Em outras palavras, a ideia de enfocar um "todo" empresarial de maneira instrumental e alheia às relações é, além de simplificadora, contraproducente, pois o ser humano realiza processos de compartilhamento de forma orgânica, genuína, diversa e afetiva.

Então, alinhado à importância de vislumbrar o conceito de organizações, superando uma lógica de controle, regularidade e uniformidade, Fausto Neto (2008) afirma a necessidade de entender tais organizações como "realidades comunicacionais" que, muito além de serem fenômenos consensuais, estão envoltas em conflitos, incertezas e dissensos que decorrem da convivência entre seres que possuem características, necessidades e motivações distintas.

Corroborando com essa perspectiva, Silvestrin e Oliveira (2015) destacam que o foco da comunicação nas organizações não deve ser apenas a eficiência de determinados processos e meios, mas sim a reflexão sobre atitudes que potencializem o convívio entre pessoas com variadas formas de agir, minimizando seu sofrimento. Ainda segundo os autores, é preciso elaborar teorias que pautem as práticas do dia a dia organizacional e que reconheçam "diferenças individuais e coletivas de uma forma mais incisiva, pois a maior violência está em garantir os direitos apenas discursivamente" (Silvestrin; Oliveira, 2015, p.42). Para isso, é preciso olhar atentamente e criticamente para a forma como cada pessoa "lê" o ambiente organizacional em diferentes situações, para os efeitos criados a partir disso, e para "que narrativas as pessoas estão articulando sobre organização, comunicação, mundo e sobre as próprias teorias" (Silvestrin; Oliveira, 2015, p.43).

No entanto, essa não é uma tarefa fácil. Rebechi e Figaro (2013) pontuam que há uma lógica hegemônica nas empresas que encara a comunicação como algo a ser planejado e avaliado de acordo com necessidades majoritariamente administrativas; esse enfoque, bastante comum na lógica da chamada "comunicação interna", se desenvolve como:

uma resposta à expectativa de empregadores e gestores em racionalizar a comunicação de maneira a estabelecer um controle social dos trabalhadores das organizações. 0 interesse principal é regular as reivindicações e as contestações sociais dos empregados, isto é, atenuar, neutralizar e tentar eliminar todo o contradiscurso que possa questionar os sistemas produtivos e as formas de administração [...]. Assim, a comunicação torna-se instrumento indispensável à legitimidade do poder. (Rebechi; Figaro, 2013, p.8-9)

Segundo as autoras, é fundamental pensar a comunicação nas empresas sem ignorar as relações de força, os conflitos e as contradições que são inerentes às realidades no trabalho. Para isso, deve-se adotar uma postura crítica e questionadora, e é nesse sentido que enxergamos o vínculo da comunicação com a aprendizagem, tomando como inspiração o pensamento freireano. Afinal, segundo Freire (1967), os sujeitos não devem ser encarados e tampouco devem se reconhecer como seres simplesmente inseridos e acomodados em um mundo preexistente, sem tensionar a realidade em que coexistem.

0 autor destaca que cada pessoa precisa ter uma verdadeira presença no mundo, e isso envolve tomar consciência de que essa presença se constitui e transforma o cotidiano a partir da tensão das heranças genéticas com as heranças sociais, culturais e históricas que se formam dia após dia (Freire, 1996). 
Para que esse processo de conscientização ocorra, Freire (1983) ressalta que é fundamental que cada ser humano seja capaz de efetuar processos de leitura. De acordo com Guaraldo (2013, p.55-57): "De forma ampla, a leitura pode ser compreendida como uma atribuição de sentidos [...] frente a qualquer forma simbólica" escrita ou não, e ainda "pode significar concepção" sobre determinado universo.

É especialmente com base nessas definições de leitura que entendemos a importância da aprendizagem, encarando-a a partir de uma perspectiva freireana. Segundo essa perspectiva, um sujeito consegue problematizar sua existência quando é capaz de conceber a realidade a partir de uma leitura de mundo crítica, ou seja, quando compreende e atribui sentidos a aspectos ligados ao contexto sócio histórico, sendo que a compreensão desses aspectos deve ser inseparável da simples leitura das palavras. Nessa mesma ordem, Guaraldo (2013, p.57) afirma: "Num movimento que vai da leitura do mundo à leitura da palavra e da palavra retorna ao mundo, está presente uma forma de ler e reescrever a realidade [...] [que] equivale a transformá-la através de uma prática consciente".

$\mathrm{Na}$ abordagem freireana, os processos de leitura de mundo são trabalhados com foco na alfabetização de adultos, mas entendemos que o objetivo é propiciar que, muito além de conhecer e utilizar palavras no dia a dia, cada sujeito possa "aprender a escrever a sua vida, como autor e como testemunha de sua história, isto é, biografar-se, existenciar-se, historicizar-se" (Fiori, 1987, p.6), e assim, integrar-se e modificar a realidade ao invés de simplesmente se ajustar a ela (Freire, 1967).

De acordo com Freire (1983), o sujeito não é capaz de realizar esse processo de construir leituras de mundo e reconhecer-se como um ser de transformação isoladamente, e para tal, depende invariavelmente de sua relação com outras pessoas, ou seja, da comunicação. 0 autor destaca: "O sujeito pensante não pode pensar sozinho; não pode pensar sem a coparticipação de outros sujeitos no ato de pensar sobre o objeto. Não há um 'penso', mas um 'pensamos'. É o 'pensamos' que estabelece 0 'penso', e não o contrário" (Freire, 1983, p.45).

Diante do exposto, defendemos que a compreensão sobre a relação inextrincável entre comunicação e aprendizagem e a necessidade de ambas para a vida humana pode gerar outras formas de encarar a inovação nas organizações de uma perspectiva transformadora, que vá além da positividade que costuma estar atrelada ao fenômeno. Trazemos algumas reflexões sobre isso na sequência.

\section{O POTENCIAL TRANSFORMADOR DA INOVAÇÃO A PARTIR DA COMUNICAÇÃO E DA APRENDIZAGEM}

Godin (2014) destaca que, apesar do entendimento de inovação ter se modificado ao longo dos séculos e dele ser um fenômeno sobre o qual podem ser atribuídos diferentes significados de acordo com o contexto, a maior parte dos atores governamentais, empresariais e midiáticos elegeu uma representação tecnológica e mercadológica dominante sobre 0 conceito, induzindo ações capitaneadas pelos empreendedores orientadas para o crescimento, o progresso, a industrialização e 0 aumento da competitividade.

Não temos dúvidas de que existem vários benefícios oriundos dessa escolha para o desenvolvimento das empresas e de diversos setores da economia, mas acreditamos que é preciso questionar também quais são as pessoas que efetivamente estão participando desse processo e usufruindo desses benefícios nas organizações, e, além disso, se as inovações criadas a partir dessa lógica estão efetivamente trazendo melhorias e avanços estruturais no dia a dia dos indivíduos das empresas, ou estão apenas contribuindo para fortalecer a hegemonia, a posição hierárquica, a visão totalizante e os privilégios de uma minoria. Em outras palavras, precisamos nos perguntar: por quem, para quem e para que estão sendo feitas as inovações nas 
organizações? Elas são um meio real para promover mudanças positivas no cotidiano da maior parte das pessoas, ou apenas um fim utilizado por alguns para justificar a manutenção de relações de poder, de ordem, de adequação e de exclusão através de novos produtos, serviços e processos? E mais: apesar de haver um discurso dominante que estimula permanentemente a inovação e a mudança nos dias de hoje, se um indivíduo da organização decidir questionar a ordem estabelecida e algumas práticas prejudiciais ao seu cotidiano na empresa, ele será valorizado, reconhecido e ouvido por essa atitude, ou será deslegitimado de maneira parecida com a que ocorria até o século XVIII?

A nosso ver, vislumbrar a inovação a partir da comunicação e da aprendizagem, indo além de um viés meramente econômico, utilitarista e unívoco, é uma forma de enxergar a essência humana e social do conceito, reconhecendo-o como um catalisador de mudanças não apenas para a diferenciação e o fortalecimento das organizações, mas também para a estruturação de condições de trabalho melhores e mais justas construídas pelas e para as pessoas que nela atuam, condições essas que se concretizem em ações reais e não apenas em discursos romantizados de valorização dos empregados que defendem a harmonização e o consenso, ao invés de reconhecer os dissensos e contradições inerentes às relações humanas.

Aliás, se os empreendedores das organizações considerarem, a partir de autores como Silvestrin e Oliveira (2015), que 0 foco da comunicação não deve ser apenas a eficiência de determinados meios e práticas, mas também o fortalecimento do convívio entre pessoas diferentes com o reconhecimento das características, narrativas e direitos diversos que elas possuem, e, adicionalmente, baseando-se em Freire (1967), que os processos de aprendizagem devem permitir que os seres humanos se integrem verdadeiramente ao mundo ao invés de simplesmente se acomodarem a um contexto preexistente, já teremos, na nossa visão, um potencial inovativo nas empresas.

Compreendemos que trilhar alguns caminhos seja importante para estabelecer essa forma diferente de encarar a inovação, como: ampliar estudos sobre o tema a partir de uma abordagem mais crítica, refletindo sobre alternativas para ressignificar essa vertente; desconstruir gradualmente algumas visões instrumentais e prescritivas sobre comunicação e aprendizagem que ainda são dominantes em muitas empresas e dificultam a realização de práticas mais dialógicas, respeitosas, colaborativas e reflexivas; e, além disso, viabilizar maneiras, nas organizações, de estimular e realizar questionamentos conscientes e coletivos sobre os valores, os discursos, as práticas e os resultados que precisam ser modificados, bem como sobre as formas que serão adotadas para promover essas mudanças.

Dito de outro modo, ao invés de enxergar a visão predominante da inovação como unanimidade e panaceia, é preciso efetuar leituras de mundo plurais, inclusivas e relacionais, de modo que elas contribuam para a criação de espaços mais abertos, justos e acolhedores nas organizações.

\section{CONSIDERAÇÕES FINAIS}

A inovação costuma ser encarada como uma palavra de ordem sumariamente ligada à tecnologia, ao desenvolvimento econômico, ao progresso e à atuação heroica do empreendedor.

A partir dessa visão ufanista, constrói-se um discurso hegemônico repetido como "verdade absoluta" sem um olhar reflexivo, que busca a manutenção de uma ordem capitalista alijada de uma crítica aprofundada.

Para nós, essa perspectiva esvazia de sentido a essência humana social, transformadora e multidimensional da inovação, encarando a capacidade de inovar como mero fim, e não como meio capaz de realizar mudanças que efetivamente melhorem o cotidiano de pessoas distintas que compõem uma organização. 
Por isso, destacamos a importância de ir além desse enfoque, e ressaltamos a necessidade de enxergar a comunicação e a aprendizagem como constituintes da inovação para que esses processos contribuam para tensionar as estruturas e práticas que minimizam a atuação dos sujeitos ao seguimento de prescrições, à busca por novidades mercadológicas utilitárias e à homogeneização de ideias, vontades e visões.

Para avançar nas reflexões que já foram feitas neste ensaio, entendemos que alguns pontos sobre a temática merecem tensionamentos e problematizações que podem ser aprofundados em outros estudos, tais como: quais são os impactos negativos que o culto ao novo e a valorização exacerbada da inovação tecnológica podem trazer às organizações e aos indivíduos? Quais são as estratégias discursivas utilizadas para legitimar a busca desenfreada pela inovação e silenciar as consequências indesejáveis desse processo? Que outras abordagens temos, tanto na área de comunicação e aprendizagem quanto em outras áreas, para oferecer olhares mais humanizados, inclusivos e complexos à inovação que estejam além do viés atual predominante? Quais são os benefícios que uma abordagem mais social e humana sobre a inovação pode trazer para o dia a dia de pessoas em diferentes níveis das empresas, e que obstáculos temos para estruturar ações nesse sentido?

Talvez, por meio dessas reflexões, seja possível delinear caminhos que contribuam para ampliar a capacidade dos diferentes indivíduos da organização, não apenas para que eles se ajustem ao contexto, mas também para que eles atuem transformando-0, modificando-o, ou, em outras palavras, inovando-o.

\section{REFERÊNCIAS}

BADILLO, Patrick Yves. Les théories de l'innovation revisitées: une lecture communicationnelle et interdisciplinaire de l'innovation? Du modèle <<Émetteur >> au modèle communicationnel. Les Enjeux de l'information et de la communication, Échirolles, n.14, p.19-34, 2013. Disponível em: www.cairn.info/revue-les-enjeux-de-l-information-et-de-la-communication2013-1-page-19.htm. Acesso em: 15 ago. 2015.

CARNEIRO, Mara. Mitos e verdades sobre inovação \#1 - é meio e não fim. Insights and Thoughts, [São Paulo], 13 abr. 2013. Disponível em: https://insightsandthoughts.com.br/2016/04/13/mitos-e-verdades-sobre-inovacao-1-e-meio-nao-fim/. Acesso em 26 jul. 2019.

D'ALMEIDA, Nicole; ANDONOVA, Yanita A comunicação das organizações. Anuário Unesco/Metodista de Comunicação Regional, São Paulo, v.12, n.12, p.31-42, 2008.

DIAZ-ISENRATH, Maria Cecília. Máquinas de pesquisa: o estatuto do saber no capitalismo informacional. 2008. Tese (Doutorado em Ciências Sociais) - Universidade Estadual de Campinas, Campinas, 2008.

FAGERBERG, Jan. Innovation: a guide to the literature. Oslo: University of Oslo, 2003. Disponivel em: https://smartech.gatech. edu/bitstream/handle/1853/43180/JanFagerberg_1.pdf?sequence=1\&isAllowed=y. Acesso em: 5 dez. 2019.

FAUSTO NETO, Antônio. Comunicação das organizações: da vigilância aos pontos de fuga. In: OLIVEIRA, Ivone de Lourdes; SOARES. Ana Thereza Nogueira. Interfaces e tendências da comunicação: no contexto das organizações. São Caetano do Sul: Difusão, 2008. p.39-64.

FIORI, Ernani Maria. Prefácio. In: FREIRE, Paulo. Pedagogia do oprimido. 17.ed. Rio de Janeiro: Paz e Terra, 1987. 
FONTENELLE, Isleide Arruda. Para uma crítica ao discurso da inovação: saber e controle no capitalismo do conhecimento. Revista de Administração de Empresas, São Paulo, v. 52, n. 1, p. 100-108, 2012.

FREIRE, Paulo. Educação como prática da liberdade. Rio de Janeiro: Paz e Terra, 1967.

FREIRE, Paulo. Extensão ou comunicação?7. ed. Rio de Janeiro: Paz e Terra, 1983.

FREIRE, Paulo. Pedagogia da autonomia: saberes necessários à prática educativa. São Paulo: Paz e Terra, 1996.

GODIN, Benoit. Innovation: the history of a category. Project on the Intellectual History of Innovation, Montréal, n. 1, 2008. Disponível em: http://www.csiic.ca/PDF/IntellectualNo1.pdf. Acesso em: 4 out. 2015.

GODIN, Benoît. The vocabulary of innovation: a lexicon. Project on the Intellectual History of Innovation, Montréal, n.20, 2014. Disponível em: http://www.csiic.ca/PDF/LexiconPaperNo20.pdf. Acesso em: 10 out. 2015.

GUARALDO, Tamara de Souza Brandão. Práticas de informação e leitura: mediação e apropriação da informação nas cartas de leitores de um jornal popular do interior de São Paulo. 2013. Tese (Doutorado em Ciência da Informação) - Faculdade de Filosofia e Ciências, Universidade Estadual Paulista Júlio de Mesquita Filho, Marília, 2013.

JUCEVIČIUS, Giedrius. Innovation culture: the contestable universality of the concept. Social Sciences, [s.l.], v.4, n.58, p.7-19, 2007.

JUCEVIČIUS, Giedrius. Culture vs. cultures of innovation: conceptual framework and parameters for assessment. In: INTERNATIONAL CONFERENCE ON INTELLECTUAL MANAGEMENT \& ORGANISATIONAL LEARNING, 7., Hung Hom Bay. Proceedings [...]. Hung Hom Bay: The Hong Kong Polytechnic University, 2010. p.236-244.

MARCHIORI, Marlene (org.). Comunicação em interface com cultura. Rio de Janeiro: Senac, 2013.

MESSINA, Graciela. Mudança e inovação educacional: notas para reflexão. Cadernos de Pesquisa, São Paulo, n.114, 2001. Disponível em: http://www.scielo.br/pdf/cp/n114/a10n114.pdf. Acesso em: 4 dez. 2019.

REBECHI, Claudia Nociolini; FIGARO, Roseli. A comunicação no mundo do trabalho e a comunicação da organização: duas dimensões distintas. Animus, Santa Maria, v.12, n.23, p.1-21, 2013.

SCHUMPETER, Joseph Alois. Teoria do desenvolvimento econômico: uma investigação sobre lucro, capital, crédito, juros e o ciclo econômico. São Paulo: Nova Cultural, 1997.

SERRES, Michel. Hominescências: o começo de uma outra humanidade. Rio de Janeiro: Bertrand Brasil, 2003.

SIEVERS, Burkard. 'It is new, and has to be done!': socio-analytic thoughts on betrayal and cynicism in organizational transformation. Culture and Organization, [s.l.], v.13, n.1, p.1-21, 2007.

SILVESTRIN, Celsi Brönstrup.; OLIVEIRA, Jair Antonio de. Implicações sociais e políticas da comunicação organizacional: para quem é a teoria? In: SCROFERNEKER, Cleusa Maria Andrade. (org.). De qual comunicação organizacional estamos falando? Porto Alegre: EDIPUCRS, 2015. p.39-50. 
SPINK, Peter Kevin. O resgate da parte. Revista de Administração, São Paulo, v.26, n.2, p.22-31, 1991.

SPINK, Peter Kevin. A Organização como fenômeno psicossocial: notas para uma redefinição de psicologia do trabalho. Psicologia \& Sociedade, Belo Horizonte, v.8, n.1, p.174-192, 1996.

STOFFREGEN, Diana Cardona. Principios estratégicos para la intervención. Metacomunicación, [s.l.], v.3, n.5, p. 8-27, 2014. Disponível em: https://revistametacomunicacion.files.wordpress.com/2011/10/mt6-art1.pdf. Acesso em: 4 dez. 2019.

Artigo recebido em 31.07.2019 e aprovado em 13.12.2019. 